\title{
The Invisible Universe
}

\section{Michael Rowan-Robinson}

Abstract. With our own eyes we can see the night sky of the stars, planets and the Milky Way, the arena of pre-telescopic astronomy. Modern optical telescopes have opened up the universe of galaxies and we are familiar with the superb images of the Hubble Space Telescope. But with the invisible wavelengths of radio, infrared and X-ray, a very different universe comes into view. The astronomy of the invisible wavelengths was inaugurated by William Herschel in 1800 but developed very slowly over the next 160 years. The past fifty years have seen an explosion in our understanding of this strange world.

Our imagination, and our ability to visualize the night sky and the universe, is strongly conditioned by our senses, especially vision. The night sky has had a profound influence on our image of ourselves, how we see ourselves within this landscape. This is strongly reflected in literature and the arts. But when we look to see who talks most about the night sky, the stars, one writer dwarfs all others and that is Shakespeare. In 'Othello' (2.1.13-15) He makes it clear that he was extremely familiar with the night sky, and perhaps was even in touch with the new ideas of Copernicus and Tycho Brahe.

The wind-shak'd surge, with high and monstrous mane

Seemed to cast water on the burning Bear,

And quench the guards of th'ever fixed pole.

The violence of the storm encountered by Othello on his way to Cyprus are indicated by the fact that the Great Bear, which never sets from UK latitudes, seems to disappear below the waves (amusingly, not quite such a good metaphor at the latitude of Cyprus). In 'Henry IV', part 1 (2.1.1-3) Shakespeare has the porters in the inn-yard realise they are late from the position of the Wain, or Plough, in the sky:

Heigh-ho! An't be not four by the day,

I'll be hanged; Charles' Wain is over the new chimney

And yet our horse not packed.

Michael Rowan-Robinson, 'The Invisible Universe', eds. Nicholas Campion and Rolf Sinclair, Culture and Cosmos, Vol. 16 nos. 1 and 2, 2012, pp. 255-259. www.CultureAndCosmos.org 
256 The Invisible Universe

Shakespeare's Julius Caesar (3.1.60) says 'I am constant as the northern star', indicating that Shakespeare knew that the night-sky rotates about Polaris, the Northern Star, although Caesar himself is unlikely to have said this because in his time as, due to the precession of the equinoxes, the pole was not particularly near Polaris. However, although many writers talk about 'the stars', rather few demonstrate that they are familiar with more than one or two. Apart from Shakespeare, other writers who show that they are familiar with the night sky include John Milton, Alfred Tennyson and Thomas Hardy. From the opening pages of chapter 2 of 'Far from the Madding Crowd', written on 1874:

The North Star was directly in the wind's eye, and since evening the Bear had swung round it outwardly to the east, till he was now at a right angle with the meridian. A difference of colour in the stars - oftener read of than seen in England - was readily perceptible here. The kingly brilliancy of Sirius pierced the eye with a steely glitter, the star called Capella was yellow, Aldebaran and Betelgeux shone with a fiery red.

To persons standing alone on a hill during a clear midnight such as this, the roll of the world eastward is almost a palpable movement. ${ }^{1}$

And one of Hardy's novels 'Two in a Tower', is actually about astronomers, and he consulted Greenwich Observatory about some of the details.

In the twentieth century two writers who were fairly obsessed by astronomy were the Russian poet, Osip Mandelstaum and the Italian novelist and poet, Italo Calvino. Now from Galileo onwards, and especially after William Herschel, astronomy ceased to be about what we can see with the naked eye, but was about what we see with telescopes. And what Herschel saw in his telescopes was evolution: stars are born in clouds of gas, they live and then they die. Byron, who visited Herschel and was shown his telescopes, grasped this remarkably clearly in his great poem 'Darkness', in which he painted a searing picture of the last survivors on earth as the sun dies:

I had a dream, which was not all a dream.

The bright sun was extinguished, and the stars

1 Thomas Hardy, Far from the Madding Crowd (New York: Henry Holt and Company, 1874), p. 9.

Culture and Cosmos 
Did wander darkling in the eternal space,

Rayless, and pathless, and the icy earth

Swung blind and blackening in the moonless air... ${ }^{2}$

One of the first to grasp the significance of the new realm of the nebulae being opened up by the Herschels and Lord Rosse was Vincent van Gogh, with his visionary painting 'Starry Night', which featured the spiral galaxy Messier 51, the Whirlpool, based on Rosse's sketch, at the centre of the vision. Today we have become familiar with the images of distant galaxies from the great optical telescopes, culminating in the iconic images from the Hubble Space Telescope. So we have digested the fact that our picture of the night sky may need to be enlarged by a telescope to get the full experience.

What I want to talk about today is the way that astronomer's vision of the universe has undergone a further complete transformation, way beyond the enlargement of vision that the optical telescope brought, through the impact of invisible radiation. ${ }^{3}$ This revolution was initiated in 1800 by William Herschel, when he used a prism and a thermometer to show that there were invisible rays beyond the red end of the spectrum. In a series of almost 200 experiments he demonstrated that these invisible rays behaved exactly like visible light and that they were the same phenomenon as radiant heat. Herschel had launched infrared astronomy but it took a hundred years for the first reliable detections of even the brightest stars. It was not till the 1960s that infrared astronomy really took off, with the discovery of sources by the Two-Micron Sky Survey that were strong in the infrared but invisible at optical wavelengths. These were followed up during the 1970s from the ground and from balloon and airborne telescopes.

Clerk Maxwell had shown in 1876 that optical and infrared light were simply electromagnetic waves, with the wavelength of the waves determining the colour of the light, with the visible spectrum confined to the narrow range of wavelength 0-4-0.7 microns. Soon afterwards Hertz

2 Lord Byron, 'Darkness', lines 1-5, at http://www.online-literature.com/byron/685/.

3 For the history of modern cosmology in what follows, see Norris D Hetherington, The Encyclopaedia of Cosmology: Historical, Philosophical and Scientific Foundations of Modern Cosmology (New York and London: Garland Publishing, 1993). 
258 The Invisible Universe

detected radio waves and Roentgen discovered X-rays. Suddenly there was this whole vast spectrum of electromagnetic radiation.

The first of the invisible wavelengths to take off was radio. Karl Jansky detected radio noise from the Milky Way in 1936 - as the New Yorker is reported to have sardonically put it 'this was probably the furthest anyone had ever gone looking for trouble'. Astronomers didn't take much notice of Jansky's discovery and it fell to the amateur Grote Reber to make the next step, mapping the Milky Way in the 1940s. John Hey discovered radio emission from the Sun during the War, and after the War found the first discrete sources. Surveys at Cambridge and in Australia found hundreds more and it was soon clear that most of these were distant galaxies. The discovery of quasars followed in 1964 and over the next decades the realization that radio-galaxies and quasars are powered by massive black holes in galactic nuclei. Pulsars, pulsating radio sources associated with neutron stars, were discovered in 1967.

Meanwhile X-ray astronomy had also emerged, at first using rockets to detect X-rays from the Sun and then from an unexpectedly bright compact source Sco X-1. The Uhuru satellite in 1976 discovered dozens of compact X-ray sources associated with white dwarfs, neutron stars and stellar-mass black holes. Uhuru also detected very hot, billiondegree, gas in clusters of galaxies, a few extragalactic sources, and the Xray background radiation.

The cosmic microwave background radiation, relic of the hot Big Bang, was found in 1965, and shown to have a $2.7 \mathrm{~K}$ black-body spectrum over the next decade. The 1970s saw the emergence of ground-based submilllimetre astronomy, confined at first to bright radio sources, but gradually opening up the study of dusty star-forming galaxies. So the 1960s and 70s saw the emergence of all the main astronomies of the invisible wavelengths. The subsequent decades saw the development of wonderful space missions - IRAS, ISO, Spitzer, COBE, WMAP, Einstein, Chandra, XMM - and ground-based telescopes - UKIRT, JCMT, CFHT, CSO, IRAM, to name a few. For over thirty years now we have worked in an era of astronomy across the whole electromagnetic spectrum.

Is this reflected in the literature and arts of our era? With a few exceptions, I think not. I think we are still fixated on the visible night sky and on optical images. And there is a problem here of how our imagination works. To visualize discoveries at the invisible wavelengths we generate an image that looks as much like an optical image as possible, using false colour. Sometimes the colour is used to represent 
intensity and in the early days the radio-astronomers used some very garish colour tables. Gradually more soothing versions have been developed, typically using quite a narrow palette of colour. The more illuminating false-colour images represent different wavelengths with different colours, with blue denoting shorter wavelengths ('hotter') and red denoting longer wavelengths ('colder').

Do we have any alternative to this way of representing the invisible universe? Can we enlist any other sense than our eyes? Well our skin does detect infrared radiation and we can sense the Sun of course, but also another human being, radiating about 100 Watts of energy, at a distance of about 1 metre. This doesn't take us very far in sensing the infrared landscape. The rattlesnake does a bit better with its infrared eye, but this is a pretty crude device too.

I don't think smell offers many options, though our ability to detect a wide range of chemical flavours does have a nice analogy with spectroscopy, where we use spectral lines to identify atoms and molecules in cosmic objects. Sound has some possibilities. There are some nice audio recordings of pulsars, some of which have quite a catchy beat. The cosmic microwave background can be seen as white noise on a TV screen or again as an audio signal. It sounds pretty much like noise but this is not a very good representation. The fluctuations in the cosmic microwave background have a beautiful harmonic structure, with a strong fundamental and a series of overtones. If these were transcribed for a synthesizer it would sound rather majestic. These fluctuations really are the acoustic echo of the Big Bang itself, so an audio representation is very appropriate. It gives the answer to a question my son asked me when he was four: 'How loud was the Big Bang?'. Very loud but sonorous.

So there is not much alternative to trying to represent the invisible universe in visual terms. Perhaps we need to be more imaginative with the colour tables, not always striving for something that looks pretty. We need to accompany these images with words that convey the otherness of the invisible. We have to think of the ground-based telescopes and space missions as extensions of our own eyes. Planck and Herschel are up there now mapping the submillimetre sky. They represent human eyes searching. What comes down is not immediately a picture, but we struggle to make sense of it and to reach a picture. 